\title{
Enquête
}

Archives de la revue Enquête

5 | 1989

Biographie et cycle de vie

\section{L'éducation comme projet de vie des femmes de la nouvelle classe moyenne}

Jean-Pierre Roos

\section{(2) OpenEdition}

Journals

Édition électronique

URL : http://journals.openedition.org/enquete/106

DOI : 10.4000/enquete.106

ISSN : 1953-809X

Éditeur :

Cercom, Éditions Parenthèses

Édition imprimée

Date de publication : 2 mars 1989

\section{Référence électronique}

Jean-Pierre Roos, «L'éducation comme projet de vie des femmes de la nouvelle classe moyenne »,

Enquête [En ligne], 5| 1989, mis en ligne le 27 juin 2013, consulté le 20 avril 2019. URL : http:// journals.openedition.org/enquete/106 ; DOI : 10.4000/enquete.106

Ce document a été généré automatiquement le 20 avril 2019 


\title{
L'éducation comme projet de vie des femmes de la nouvelle classe moyenne
}

\author{
Jean-Pierre Roos
}

Je veux remercier Martine Burgos qui m'a beaucoup aidé en ce qui concerne la forme et le contenu du texte.

1 Il existe un quasi-consensus sur le fait que quelque chose s'est passé avec les valeurs traditionnelles du travail dans les sociétés développées et industrialisées - et surtout dans les pays où l'éthique protestante du travail a été en vigueur, comme dans les pays nordiques. Dans les pays méditerranéens on peut se demander si cette éthique du travail a jamais été importante, sauf parmi des minorités très modernes ${ }^{1}$. Dans les pays nordiques, comme en Finlande, il n'est pas question de mettre en doute l'idée que notre éthique du travail ne soit, depuis longtemps, profondément «protestante». Historiquement, on peut quand même noter que la diffusion de cette éthique parmi les paysans est assez récente, et, encore au début du siècle, on les décrivait fréquemment comme très paresseux, peu sérieux, sans loyauté. Dans les contes populaires, en revanche, les personnages rusés l'emportaient toujours sur les bons travailleurs stupides. Ainsi dans les pays comme la Finlande, il serait plus judicieux de parler d'un développement très rapide et récent d'une éthique du travail protestante, sur quelques décennies, parmi les premiers entrepreneurs bourgeois dans le milieu du XIX ${ }^{e}$ siècle. Puis elle se répand, peu à peu, grâce aux mouvements antialcooliques et plus tard ouvriers, parmi les ouvriers et les petits paysans radicaux ${ }^{2}$.

2 Si l'on peut, actuellement, parler d'un recul de l'éthique du travail, il s'agit d'un phénomène très récent. Quand j'ai étudié les récits de vie des personnes nées en ce siècle, j'ai pu constater que, pour les personnes âgées d'environ 40 ans, le rôle du travail a déjà beaucoup changé. On en parle assez peu, on n'envisage plus que la vie soit une vie de travail dur et constant, certes on se situe dans le récit à travers l'évocation de sa profession, de sa carrière, mais le travail comme travail est très peu présent. Par contre, 
on commence à parler d'école, des décisions concernant l'éducation, de la vie d'étudiant, etc. ${ }^{3}$.

Quand j'ai remarqué que ce phénomène était encore plus accentué dans ma toute dernière série de récits de vie $^{4}$, et surtout parmi les femmes, j'ai commencé à m'interroger sur ses causes. Est-ce que l'éthique du travail serait remplacée par une éthique d'éducation et d'activités auxiliaires, dont la nature serait totalement différente? $\mathrm{Ou}$ bien l'éthique du travail se présente-t-elle simplement comme une éthique d'éducation, mais avec les mêmes fonctions : contrôle de vie, succès personnel, et donc sans changement essentiel des mentalités? Dans ce dernier cas, cela signifierait que la plupart des débats portant sur la perte d'une éthique « protestante » du travail reposent simplement sur une erreur d'analyse.

4 La réponse que je proposerai, à la suite d'un examen approfondi des récits de vie, est que l'éthique de l'éducation est bien en partie de même nature que celle du travail, mais qu'elle comporte aussi d'autres éléments intéressants, comme par exemple le désir d'exercer un contrôle sur sa propre vie, non pas dans un sens ascétique, mais avec l'idée qu'il existe des solutions de rechange ouvertes en tout temps, et qu'un choix est toujours possible. Ce qui implique, bien sûr, une stratégie de réussite (au sens de P. Bourdieu), comportement assez spécifique à la classe moyenne. L'éducation peut également être conçue comme une activité importante en soi, comme un besoin.

\section{L'approche par les récits de vie}

L'intérêt de l'utilisation des récits de vie en sociologie est suffisamment établi pour qu'il ne soit pas nécessaire de discuter les avantages ou désavantages de cette approche ${ }^{5}$. À mon avis, la chose un peu méconnue est que les récits de vie sont justement des récits, des narrations ${ }^{6}$, avec des règles structurales bien précises de présentation où le rôle du narrateur est prépondérant, car il a le monopole en ce qui concerne l'intrigue et le point de vue. Comme l'écrit Lalive d'Épinay : «Le récit de vie est doublement subjectif puisqu'il s'agit de la vie construite d'une personne puis il est interprété à un moment donné de cette vie, dans une situation précise, par cette même personne ${ }^{7}$. "

Il est évident que les récits de vie ne peuvent être utilisés partout et quel que soit le problème, mais ils présentent un grand intérêt lorsqu'il s'agit de comprendre comment les gens construisent et comprennent leur vie. Selon moi, le concept d'habitus, développé par Bourdieu ${ }^{8}$ est un concept idéal dans le cadre des récits de vie. En présentant sa vie, l'homme présente surtout les points pertinents pour l'entendement de son habitus (d'un autre côté, certains corollaires de l'habitus sont moins présents comme les choix concrets, les goûts...). Quand il s'agit, en particulier, d'un changement de l'habitus, le récit de vie peut nous donner des clés très précises pour saisir la différence entre l'ancien et le nouvel habitus. Normalement, celle-ci n'est pas observable. Dans certains cas, les récits de vie peuvent cacher soit l'un ou l'autre aspect de l'habitus, mais le plus souvent les deux habitus s'expriment comme un point central du récit. Il est également intéressant de remarquer que les règles narratives, les dispositifs structuraux d'un récit particulier, peuvent nous aider dans l'analyse des différents habitus. La structure du récit, les causes et les intentions peuvent changer de manière dramatique. Une analyse de ce type de récit de vie peut aussi aboutir à la constatation qu'un des habitus doit être considéré comme 
dominant dans le récit tandis que l'autre habitus - important, parce que c'est l'habitus sans lequel on ne peut pas agir dans la position actuelle - n'est pas dans l'économie narrative9.

\section{Les concours de récits de vie}

7 Le matériau que j'utilise ici a été réuni lors d'un concours organisé par les trois syndicats finlandais d'employés, qui réunissent une grande majorité des salariés non ouvriers des secteurs public et privé. Le concours était annoncé dans les journaux des syndicats et dans les grands quotidiens. L'annonce était très générale : on demandait seulement de fournir une autobiographie (récit de vie écrit par l'auteur), sincère, sans exigence particulière en ce qui concerne le style, la façon d'écrire, la longueur (environ cinquante pages maximum). On indiquait aussi que les textes seraient utilisés comme matériau pour la recherche. Tout participant devait donc donner son accord sur les conditions d'utilisation.

8 Plus de trois cents récits de vie nous sont parvenus, la plupart dactylographiés, environ 12000 pages au total. Quelque deux cents provenaient de femmes, ce qui reflète à peu près la répartition des sexes dans les syndicats. Presque toutes étaient nées pendant et après la Seconde Guerre mondiale, elles avaient donc moins de 45 ans au moment du concours. Dans cet exposé, il sera surtout question des femmes de moins de 45 ans, employées, infirmières et enseignantes, qui peuvent être considérées comme représentatives de la nouvelle classe moyenne. Elles habitent les grandes villes et sont le plus souvent finlandaises, mariées avec des hommes de la même classe.

\section{La nouvelle classe moyenne finlandaise}

9 La nouvelle classe moyenne est un concept très utilisé et très discuté depuis quelques années. On en a proposé plusieurs définitions : les nouvelles classes moyennes seraient des employés de l'État-providence : travailleurs sociaux, enseignants, employés dans le secteur de la santé; ou bien les cadres supérieurs et moyens des entreprises ${ }^{10}$, ou encore des fractions de la classe dominante en déclin qui chercheraient des secteurs d'activité pour augmenter leur capital social (les nouveaux petits-bourgeois de Bourdieu). Dans la plupart des cas ce sont des gens issus des milieux petits-bourgeois ou de la classe moyenne, mais qui ont dû trouver de nouvelles manières de se définir et de se comprendre. L'analyse de ces nouvelles classes ou couches sociales s'effectue à partir de ces façons de se voir et de se représenter: sociabilité, relation au travail et au loisir, volonté de créer de nouveaux espaces sociaux où elles dominent, etc.

10 En Finlande, la nouvelle classe moyenne possède la majorité des caractéristiques qui sont évoquées dans les textes cités précédemment, mais elle a quelques traits spécifiques que je pense avoir dégagés dans mes travaux. Le fait le plus important est qu'en Finlande la plupart des personnes des nouvelles classes moyennes sont issues des classes populaires. Pour Catherine Bidou ${ }^{11}$, par exemple, les nouvelles classes moyennes sont une sorte de point de rencontre de gens venant d'en haut et de gens venant d'en bas, mais il est évident que le « milieu » l'emporte. En Finlande, très rares sont les cas des personnes de ces couches qui soient issues de la bourgeoisie et peu nombreuses celles qui proviennent de la petite bourgeoisie (je distingue la petite paysannerie de la petite bourgeoisie). Le plus grand nombre vient de la campagne ou de familles ouvrières des villes moyennes, 
ayant un mode de vie presque campagnard. Ainsi, une observation intéressante dans les nouvelles classes moyennes consiste justement dans la transformation des habitus, des ressources et modes de vie qu'ils ont connus pendant leur trajectoire ${ }^{12}$.

11 En outre, les récits de vie ont montré l'évolution des projets et des intérêts de la nouvelle classe moyenne, ce qui permet de compléter l'image de cette classe. On constate, bien sûr, un effet de représentation typiquement bourgeois, mais ce qui est très frappant chez les membres de la nouvelle classe moyenne, c'est l'ambivalence consciente de leurs projets, combinée avec une forte envie de contrôler leur vie jusqu'au bout : l'ambivalence et aussi le désir de disposer constamment de plusieurs solutions de rechange et même le choix de suivre deux voies en même temps ${ }^{13}$. Beaucoup des caractéristiques évoquées se rencontrent chez les femmes de classe moyenne. En fait, il me semble que la nouvelle classe moyenne est plutôt de type "féminin ", que ce sont les femmes qui intériorisent le plus les propriétés de cet ensemble.

\section{Récits de vie des femmes de la génération d'avant- guerre}

Dans un travail antérieur, j'ai présenté et analysé les récits de vie de plusieurs générations en Finlande ${ }^{14}$. Ces générations diffèrent notamment par la valeur que prend le travail et par la nature du travail présenté. Les femmes des générations antérieures racontent très souvent une vie totalement vouée au travail. Travail à la maison, avec les enfants, travail hors maison, mais toujours le travail. L'école joue un rôle minime dans ces récits. La relation de ces femmes à leur travail est une relation de nécessité : c'est un travail sans questionnement, sans mise en cause. Il faut travailler, c'est normal. Ce n'est pas du tout une question d'héroïsme (ou très peu), ni même de réussite. Les femmes font des récits de vie où le travail est un sujet en soi, pas simplement une toile de fond sur laquelle se détacheraient des exploits, des aventures, c'est une réalité à laquelle on a consacré toute sa vie. Le récit infiltré par une véritable éthique du travail, dans le sens protestant du terme.

13 C'est justement cette sorte de description qui disparait complètement dans les récits des femmes nées à partir des années 1940 : le travail ne figure plus dans les récits de vie comme valeur en soi. Plus question de glorifier le travail au foyer, ou de s'attarder à une description du travail pénible en dehors du foyer. Certes, il faut travailler dur pour accéder à un logement, par exemple, mais ces exploits ne sont pas, en soi, très centraux.

Deux choses sont beaucoup plus centrales dans les descriptions des femmes de cette génération. D'abord, leurs relations interpersonnelles, à commencer par le père, jusqu'aux enfants, amants, etc. Ensuite, leurs études. Les deux s'interpénètrent parce que les études ont une grande influence sur la vie de la famille et introduisent de nouveaux personnages dans leur vie, etc. Je veux ici me concentrer surtout sur les histoires d'éducation pour en dégager la signification.

\section{Les femmes de la nouvelle classe moyenne}

15 Comme dans tous les pays développés, il existe, en Finlande, une ségrégation forte entre les occupations féminines et masculines. La classification socioprofessionnelle de la classe moyenne en subit, à un haut degré, les effets (sauf peut-être quelques professions, les 
médecins, par exemple). Les femmes de la classe moyenne (employées de bureau, travailleuses sociales, institutrices, infirmières, animatrices culturelles, etc.) occupent généralement les positions les plus basses, mais avec un capital culturel souvent bien supérieur à ce qui est demandé pour la position. Les femmes ont donc consenti dans leurs métiers mal rémunérés et peu appréciés (comme c'est le cas des travailleurs sociaux par exemple) à des investissements culturels assez importants. Il est donc plutôt normal que les femmes des classes moyennes aient un niveau d'instruction/scolarisation plus haut que celui de leurs maris.

\section{Le rôle de l'éducation}

Dans cette situation, on peut se demander quelle est la signification de l'éducation pour les femmes de la classe moyenne, comment elles l'utilisent, quelles sont leurs stratégies? À la limite, l'éducation peut être considérée comme un simple moyen pour accéder à une position désirée. Mais à l'autre extrême, elle peut être considérée comme un besoin inné et inexplicable, comme un motif pour agir. Les deux positions et leurs combinaisons sont présentes dans ces récits de vie des femmes. Une autre distinction importante consiste dans la valeur accordée à l'instruction. Nous pouvons avoir un récit de la période des études qui présente ces dernières comme un travail, un «boulot». Le point central est alors qu'on a commencé à travailler dur et bien, consciencieusement. Nous avons aussi, à l'opposé, des récits qui présentent comme une époque de loisir, d'épanouissement de soi dans la liberté et l'autonomie. Dans ce dernier cas, le sens du récit est radicalement transformé. Tandis qu'un récit de travail sur un mode héroïque est un type assez masculin - et traditionnel - de récit de vie, un récit où l'intrigue ne se déroule pas en fonction du travail, mais en fonction de relations interpersonnelles, des loisirs, des activités quotidiennes, est beaucoup plus rare et plutôt « féminin ».

En fait, il y a une stratégie narrative spécifique pour les deux cas: si l'éducation est présentée comme un moyen, elle peut être utilisée dans une chaîne d'explications causales, donc dans une narration du type que je viens de définir ; mais, si l'éducation est présentée dans le récit comme une valeur en soi, alors elle est comparable avec le mariage, la naissance d'un enfant, c'est-à-dire avec les événements qui sont les nœuds d'un récit de vie. On ne les explique pas. En revanche, dans les cas où l'on étudie et passe des examens afin d'accéder à une position ou d'obtenir une compétence, la période décrite s'insère dans le récit comme une action narrative avec sa structure propre.

\section{Premier exemple : l'éducation comme travail héroöque}

Cette femme de 39 ans a six enfants, chose très exceptionnelle ; elle est professeur dans une école professionnelle et elle a toujours fait des études en plus du travail et des enfants. C'est donc un récit héroïque, où tout est dominé par le travail : travail à l'école, travail avec les enfants, travail professionnel et, en plus, travail dans la ferme.

Tout cela commence, bien sûr, dans un village, chez des paysans moyens. À la maison, les enfants apprennent à travailler. L'école n'intéresse pas énormément la jeune fille et elle trouve un emploi après avoir terminé l'école obligatoire. Deux ans dans un bureau suffisent: elle décide de continuer sa scolarisation afin de trouver du travail plus intéressant. Au lycée elle envisage la carrière de professeur, puis entre à l'université.

Un mariage et un premier enfant mettent fin à des études à plein-temps. Elle trouve un emploi dans une école professionnelle. Pour avoir un poste plus qualifié, elle 
reprend ses études. À cette fin, elle déménage à la ville voisine avec son deuxième enfant. Pendant plusieurs mois, elle est seule avec l'enfant, fait des études très difficiles, mais avec les résultats envisagés. Ce premier obstacle franchi, elle continue les études, cette fois en se rendant à la ville universitaire voisine quelques fois par semaine. Travail le jour, les enfants le soir et le matin, études pendant la nuit: c'est comme ça qu'elle décrit sa vie quotidienne pendant plusieurs années, avec les interruptions d'études de temps en temps. Un nouvel enfant signifie aussi la possibilité d'étudier pendant les congés maternité ; « étudier semble être un virus incurable, pour elle » (elle écrit à la troisième personne).

Son mari, un technicien, achète une exploitation agricole. Elle essaie de s'occuper de bétail, mais renonce après quelque temps. Il est évident que son origine familiale, qu'elle ne mentionne qu'en passant, facilite ces essais.

Pour elle, la vie est bien compartimentée : le travail, dont elle parle peu, fonctionne plutôt comme mobile pour les études, mais sans intérêt en soi ; les études sont pour elle la vraie vocation. De sa famille et surtout ses enfants, elle ne parle que très peu. Le seul enfant mentionné individuellement est celui qui l'accompagnait à la ville universitaire.

19 C'est donc typiquement un récit du travail héroïque, où la famille ne figure qu'en toile de fond, un récit beaucoup plus typique des hommes que des femmes. Il est étonnant que la forme du récit soit très naïve et maladroite, malgré le fait qu'elle enseigne la langue finnoise.

\section{Deuxième exemple : une passionnée d'éducation}

20 Cette histoire, un peu extrême, illustre bien la passion pour l'éducation que l'on rencontre dans plusieurs autobiographies. Il s'agit d'un professeur du niveau secondaire, 44 ans, deux enfants, et qui a fait des études pendant toute sa vie.

Elle est née dans une famille de la petite bourgeoisie : son père travaille comme petit chef dans l'administration forestière de l'État. Les premières années, elle vit chez ses grands-parents à la campagne. Enfant, elle a une maladie sérieuse qui entraîne plusieurs visites à l'hôpital et des traitements douloureux, mais sans suites. Pour elle, la visite à l'hôpital est aussi une expérience d'épanouissement : toute petite elle avait déjà vu la grande ville. De retour chez ses parents, dans un village lointain, et une fois à l'école, elle est déjà exceptionnelle à cause de ses expériences et de sa position dans la classe. Elle termine première de sa classe et entre au lycée. Là, sa situation s'inverse : elle vient du village et perd sa position.

En même temps, elle est l'objet des avances d'un homme de dix ans son aîné. Elle se marie immédiatement contre le gré de ses parents. Le mariage est, dès son début, mal parti, mais le divorce est impensable. Elle veut faire les études d'institutrice, mais la tentative échoue. C'est un drame, car elle avait envisagé la carrière d'institutrice depuis sa petite enfance. En consolation, elle entre à l'école de commerce. Son premier emploi est au service de la sécurité sociale, où elle travaille quelques années. Un premier enfant, un fils, est né deux ans plus tôt.

Le mariage ne marche pas très bien «le soir je me battais pour la paix du lit ». Elle veut faire autre chose, changer sa vie. Un autre enfant naît, ce qui lui fait accepter sa situation pour quelques années. Mais un matin - elle a 25 ans - elle voit l'annonce pour les nouvelles études d'instituteur. Elle présente sa candidature, contre l'opposition du mari, et est acceptée. Une nouvelle vie commence. Elle change ses vêtements, son mode de vie. "Je retrouvais ma propre estime, les jours étaient une fête, les soirs je travaillais pour ma vie.» Cette nouvelle liberté se traduit aussi en un amant plus éduqué que son mari et en une vie privée en dehors de la maison. Elle a un premier poste dans un village près du lieu de travail de l'époux. Mais bientôt elle déménage en ville, recommençant des études.

Elle mène une vie sexuelle libre tout en faisant des études, et progressant dans sa 
carrière. À la maison, c'était le statu quo : deux étrangers vivant sous le même toit. Elle veut attendre le départ de ses enfants avant de quitter son mari. Mais un autre homme rencontré par hasard change tout cela : elle tombe amoureuse, organise la séparation, déménage dans une autre ville où vit l'homme nouveau. Mais ce dernier ne veut pas laisser sa famille; la situation devient vite compliquée : elle, seule, dans une ville étrangère, dans un nouveau poste, attendant l'homme du destin. Dans cette situation, «l'étude, ma drogue éternelle, était ma seule consolation ».

21 En conclusion, cette histoire très agitée nous montre comment les études peuvent avoir en même temps plusieurs fonctions dans une histoire de vie. Ici, elles constituent d'un côté le travail et la carrière, de l'autre le symbole d'un changement total de vie. Dans les deux cas, c'est l'éthique de l'éducation qui domine toute la vie. Donc l'éthique du travail peut coexister avec une éthique différente, une éthique d'épanouissement de soi. L'éthique de l'éducation est ici d'un côté substitut de l'éthique du travail, de l'autre, une éthique de l'épanouissement personnel.

\section{Conclusion}

22 Le problème était donc de préciser le rôle de l'éducation; substitut du travail ou manifestant une nouvelle logique? L'éthique du travail a-t-elle été remplacée par une éthique de l'éducation, des activités auxiliaires, dont la nature est totalement différente, ou bien l'éthique du travail prend-elle la forme d'une éthique de l'éducation, avec les mêmes fonctions : le contrôle de sa vie, la réussite personnelle, et cela sans changement essentiel des mentalités? Ma conclusion, toute provisoire, est que l'éducation représente pour les femmes finlandaises un nouvel avatar de l'éthique traditionnelle du travail (dite protestante), je dirais même une passion du travail qui fonctionne comme une forme d'autonomisation et de libération pour les femmes. On trouve aussi d'autres éléments très intéressants, comme par exemple le désir de contrôler sa propre vie, non pas dans un sens ascétique, mais en assumant les possibilités qui s'offrent en même temps, que l'on veut pouvoir choisir ensemble, sans sacrifier l'un ou l'autre. La stratégie de la réussite, au sens de Bourdieu, devient une activité importante en soi.

En conclusion, la logique de la plupart des récits répond plus à une éthique du travail qu'à toute autre considération. J'ajouterais que, de mon point de vue, beaucoup de discussions portant sur le déclin de l'éthique protestante du travail résultent d'une erreur d'analyse : on n'a pas vu l'homologie entre travail et nouvelles activités. Mais ici, quelques questions se posent : si on parle de l'autonomie et de la liberté comme mobiles, on dévoile alors une autre relation au travail, une relation «non aliénée ", dans laquelle le but du travail ne consisterait pas seulement à offrir sa vie pour une cause extérieure (accumulation, entreprise familiale, etc.), mais où le véritable enjeu serait la réalisation de soi, l'épanouissement de soi. L'éducation introduit donc des éléments nouveaux très importants dans l'enjeu initial. 


\section{NOTES}

1. C'est un débat considérable et bien connu. Je signale une contribution récente, cf. Bo Reimer, « No Values - New Values? Youth and Postmaterialism», Scandinavian Political Studies, 11(4), 1988.

2. R. Alapuro et al., Kansa liikkeessä (Le peuple en mouvement), Helsinki, Kirjayhtymä, 1987.

3. J. P. Roos, «Life stories of social changes : four generations of Finland », International Journal of Oral History, 3, 1985, p. 179-190 ; J. P. Roos \& A. Sicinski, eds, Ways of life in Finland and Poland. Comparative Studies on Urban Populations, Aldershot, Gower, 1987, 203 p.

4. J. P. Roos, Suomalainen elämä. Tutkimus tavallisten suomalaisten elämäkerroista (Finnish life. A study on ordinary Finns' life stories), Hämeenlinna, SKS, 1987, 254 p.

5. Cf. par exemple: D. Bertaux, Histoires de vie ou récits de pratiques? Méthodologie de l'approche biographique en sociologie, Paris, Rapport CORDES, 1976; C. Lalive d'Épinay, "Récit de vie et connaissance scientifique ", Recherches sociologiques, XVI (2), 1985.

6. U. Eco, Lector in fabula ou la Coopération interprétative dans les textes narratifs, Paris, Grasset, 1985, p. 140, a proposé une définition, s'inspirant de Van Dijk: «Une narration est une description d'actions qui requiert pour chaque action décrite un agent, une intention de l'agent, un état ou monde possible, un changement, avec sa cause et le propos qui le détermine. »

7. C. Lalive d'Épinay, « Récit de vie... ».

8. P. Bourdieu, Le sens pratique, Paris, Minuit, 1980, p. 87-109.

9. W. J. T. Mitchell, On narrative, Chicago, University of Chicago Press, 1981, X-270 p.

10. Voir à ce propos, C. Bidou, Les aventuriers du quotidien. Essai sur les nouvelles classes moyennes, Paris, Presses universitaires de France, 1984; et G. Benguigui \& D. Monjardet, "L'utopie gestionnaire. Les couches moyennes entre l'État et le corporatisme ", Revue française de Sociologie, 23-24, 1982, et le débat qui a suivi dans la Revue française de Sociologie, 1984.

11. C. Bidou, Les aventuriers...

12. Pour une discussion, cf. C. Bidou, Les aventuriers... ; et J.-P. Roos, Suomalainen elämä.

13. Par exemple, une vie campagnarde et une vie très urbaine, la séparation complète entre travail et vie privée; pour les femmes, l'envie d'avoir plusieurs enfants en même temps qu'une carrière très active, etc. : voir J.-P. Roos, K. Rahkonen, Att vilja leva annorlunda: På jakt efter den nya medelklassen $i$ Finland, University of Helsinki, Department of Social Policy, 1985, Research Reports 2.

14. J. P. Roos, Suomalainen elämä... 